\title{
Guest-editorial
}

\section{Special issue: Advances in medical intelligent decision support systems}

\author{
Vassilis Kodogiannis ${ }^{\mathrm{a}, *}$, Ilias Petrounias ${ }^{\mathrm{b}}$ and John Lygouras ${ }^{\mathrm{c}}$ \\ ${ }^{a}$ University of Westminster, United Kingdom \\ ${ }^{\mathrm{b}}$ The University of Manchester, United Kingdom \\ ${ }^{\mathrm{c}}$ Democritus University of Thrace, Greece
}

This special issue is concerned with Advances in Medical Decision Support Systems. Computer science contributions to the biomedical community started with the first expert systems. Since then advances have taken place in both the medical and biological domains and also in computer science technology. This means that additional automated support can be made available to these types of applications. These advances and their contribution to the biomedical community are the focus of this special issue. The application of Intelligent Decision Support Systems in medicine is the subject of considerable ongoing research, which concentrates on modelling some of the human actions or thinking processes, recognising diseases from a variety of input sources and even extends to the representation and querying of gene data from microarray databases. Five papers have been selected, after rigorous review, for this special issue. These cover two categories: the first set of papers focuses on the medical domain and mainly on classification of medical data for accurate diagnoses. The second set of papers focuses on biological data and more importantly retrieval queries from microarray data and relationships between gene products.

Computational intelligent methodologies are used for the detection of breast cancer in the paper by Geraldo Braz Junior et al. Breast cancer is the major cause

\footnotetext{
${ }^{*}$ Corresponding author. E-mail: V.Kodogiannis@westminster.ac.
}

of cancer-related deaths among the female population. It is well known that the best prevention method is the precocious diagnosis, which lessens mortality and improves the treatment. In the current study, spatial texture analysis of digital mammograms has been implemented. Texture measures have been obtained though the Getis-Ord Index, while classification has been carried out using support vector machines. In the paper by Dmitry Zinovev et al, two semi-supervised learning approaches based on low-level image features for the prediction of semantic characteristics in lung modules are presented. The adopted approaches are especially useful when for the same nodule and semantic characteristic there are different values (class labels) reflecting the difference in interpretation among radiologists. The work presented in the paper, using the Lung Image Database Consortium dataset, is one of the first attempts in the medical imaging field to solve a multi-value label classification problem. In the paper by Kodogiannis et al., a novel neuro-fuzzy model has been used in a decision support system for the identification in vitro of bacterial clinical isolates, collected from patients diagnosed with urinary tract infections, gastrointestinal and respiratory infections, using an electronic nose. The model constructs its initial rules by clustering while the final fuzzy rule base is determined by competitive learning. Both error backpropagation and recursive least squares estimation, are applied to the learning scheme. The convergence of training is faster because the leastsquares algorithm is applied to the estimation of con- 
sequence parameters of the system and backpropagation is applied only to the estimation of the premise parameters. The paper by Nantia Iakovidou et al., proposes a kernel-based method for ranking retrieval results for microarray data. In general retrieval queries in microarray databases can rank genes according either to their similarity by detecting functionally related genes, or to their importance by detecting genes with significant regulation role. Although both rankings are useful, they can be contradicting. In this study, a Webinspired kernel method for fusing the two rankings according to the user needs is adopted. Finally, the paper by Denaxas and Tjortjis focuses on the Gene Ontology for quantifying the biological relatedness between gene products and proposes two figures of merit scores for this purpose. The proposed framework relies on the Gene Ontology for integrating a priori biomedical knowledge into traditional data analysis approaches. The paper explores the impact of considering each aspect of the Gene Ontology individually for quantifying the biological relatedness between gene products.

The Guest Editors,

Dr. Vassilis Kodogiannis University of Westminster, United Kingdom

Dr. Ilias Petrounias

The University of Manchester, United Kingdom

Dr. John Lygouras

Democritus University of Thrace, Greece 